\title{
Jean-Louis Heim
}

\section{Grimaud-Hervé $\cdot$ L.-J. Boé $\cdot$ P. Mennecier $\cdot$ J. Granat $\cdot$ A. Dambricourt Malassé $\cdot$ A. Froment $\cdot$ M.-A. de Lumley}

Reçu le 30 mai 2018; accepté le 30 mai 2018

(C) Société d'Anthropologie de Paris et Lavoisier SAS 2018

Jean-Louis Heim, professeur de paléoanthropologie au Muséum national d'histoire naturelle, nous a quittés le 11 février 2018 à l'âge de 80 ans. Né le 29 décembre 1937 à Paris, Jean-Louis est le fils de Roger Heim (ancien directeur du MNHN) et de Panca Eftimiu, tous deux scientifiques de renommée internationale. Baigné dans cette atmosphère de recherche, Jean-Louis Heim suit un parcours littéraire avant de s'engager dans une voie médicale et scientifique qui le mènera à la paléoanthropologie. Marié à Patricia Soto-Heim, anthropologue spécialiste du peuplement paléo-indien et archaïque d'Amérique du Sud, ils ont deux fils, Alexandre et Sébastien.

Henri-Victor Vallois lui propose d'étudier les désormais célèbres hommes fossiles de La Ferrassie et lui confie l'ensemble des découvertes de cet abri-sous-roche situé sur la commune de Savignac-de-Miremont en Dordogne. Ce sujet sera l'objet de son doctorat soutenu en 1972, récompensé par le prix Bonnet de l'Académie des sciences.

Ses résultats seront ensuite publiés dans la collection des Archives de l'Institut de paléontologie humaine : Les Hommes fossiles de La Ferrassie (T. I : 1976 [1] et T. II : 1982 [2]). Jean-Louis Heim consacre ensuite ses travaux aux immatures découverts dans ce même gisement, travaux qui seront publiés aux Éditions de la Fondation Singer-Polignac sous le titre Les Enfants néandertaliens de La Ferrassie [3].

D. Grimaud-Hervé $(\bowtie) \cdot$ A. Dambricourt Malassé M.-A. de Lumley

UMR 7194, HNHP, département homme et environnement,

Muséum national d'histoire naturelle, Paris, France

Paris 5, France

e-mail : dominique.grimaud-herve@mnhn.fr

\section{L.-J. Boé}

UMR 5216, département de la parole et de la cognition, GIPSA-lab, Grenoble, France

P. Mennecier · J. Granat

UMR 7206, écoanthropologie et ethnobiologie,

Muséum national d'histoire naturelle, Paris, France

\section{A. Froment}

UMR 208, IRD, Muséum national d'histoire naturelle, Paris, France
Il y met déjà en évidence une croissance et un développement des Néandertaliens plus rapides que chez l'homme moderne. Ces mémoires, qui font le point sur la morphologie des restes crâniens et infracrâniens, restent une référence aujourd'hui, et les paléoanthropologues apprécient d'y trouver les informations anatomiques précises qu'il a livrées non seulement sur les Néandertaliens de La Ferrassie, mais aussi sur leurs contemporains. Proche de Germaine Henri-Martin, il a également participé aux fouilles de La Quina (Charente) (Fig. 1).

En 1974, il succède à Henri-Victor Vallois à la chaire de professeur de paléoanthropologie de l'Institut de paléontologie humaine, Fondation Prince Albert $\mathrm{I}^{\mathrm{er}}$ de Monaco, un titre et une fonction qui lui étaient chers. Lauréat de l'Académie des sciences, il sera ensuite nommé professeur au laboratoire d'anthropologie biologique du Muséum national d'histoire naturelle en 1992, institution à laquelle il était très attaché depuis les débuts de sa carrière scientifique en 1963.

Jean-Louis Heim entreprend, en 1985, une nouvelle reconstitution du crâne de La Chapelle-aux-Saints [4] anciennement restauré par Marcellin Boule en 1911, qui fera l'objet d'un documentaire (1986) largement diffusé et repris en 2008 (Les Origines du langage).

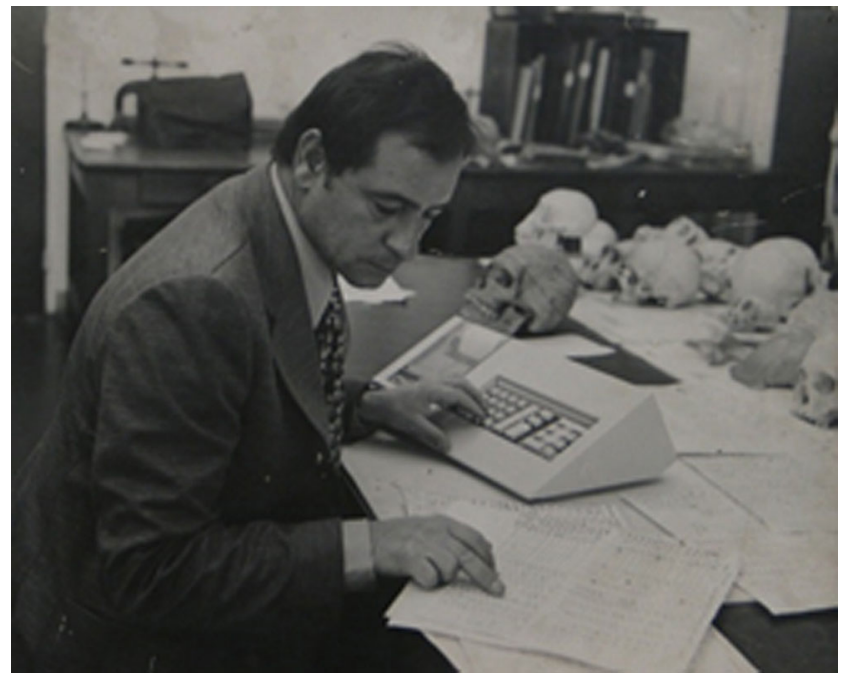

Fig.1 Jean-Louis Heim au musée de l'Homme en 1975 
Contacté en 1997 par Louis-Jean Boë et son équipe (Institut de la communication parlée, Grenoble), Jean-Louis Heim met en place un programme de recherche sur l'émergence de la parole. Le but est la reconstitution du conduit vocal de l'homme de Neandertal pour en prédire les potentialités phonatoires à partir de la localisation de l'os hyoïde par rapport à la mandibule et au rachis cervical. Jean-Louis Heim apporte à l'équipe sa profonde connaissance de la préhistoire, son érudition et une inestimable contribution en tant que paléoanthropologue. L'équipe de l'ICP élargit ses recherches à l'anthropologie et au domaine de la parole, alors que Jean-Louis Heim découvre un nouveau domaine scientifique associant sciences humaines et sciences de l'ingénieur qui seront développées lors de plusieurs projets interdisciplinaires (OHLL, OMLL, SkullSpeech). Cette collaboration scientifique va permettre d'argumenter les questions de l'acquisition et du développement du langage articulé et de son contrôle moteur à la lumière de la croissance craniofaciale. L'évolution de cette structure osseuse sera analysée par les interactions entre les éléments osseux fixes et les parties mobiles (la mandibule, l'os hyoïde et la langue). Les résultats sont une remise en cause de la théorie de la descente du larynx qui place Jean-Louis Heim et l'équipe de Grenoble au centre d'une controverse internationale. Un de leurs articles, modélisant les capacités phonatoires de Neandertal, est maintenant cité au niveau international comme une référence [5].

Son intérêt scientifique n'étant pas restreint aux espèces fossiles, Jean-Louis Heim entreprend l'étude des momies des nécropoles d'Aïn el-Labakha et de Douch dans l'oasis de Kharga en Égypte [6] et n'abandonne pas le terrain par la reprise d'anciennes fouilles de la nécropole Xiongnu de Gol Mod en Mongolie (Fig. 2).

Les résultats de ses travaux ont fait l'objet de nombreuses présentations publiques dans des colloques et des congrès au niveau national comme international, une centaine de

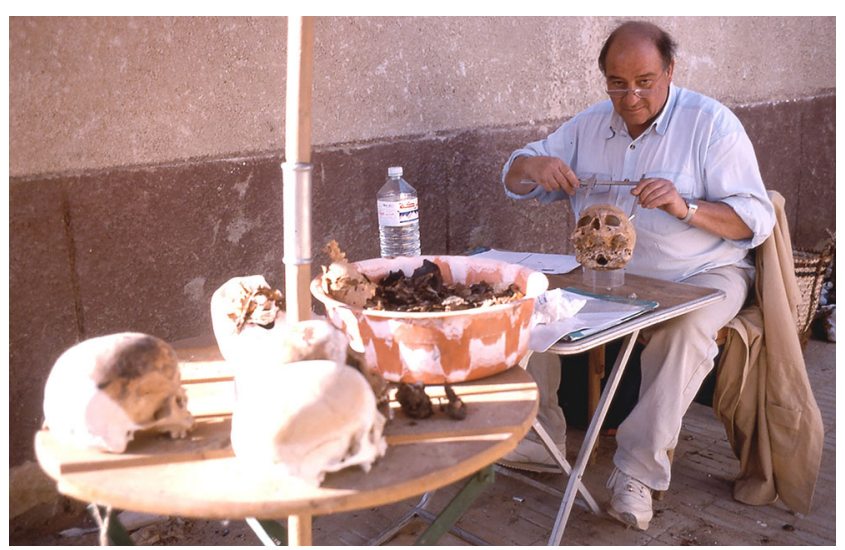

Fig.2 Jean-Louis Heim sur le site d'Aïn el-Labakha (Égypte), en 2010 publications dont quelques livres qui ont rencontré un grand succès comme 700000 siècles d'histoire humaine aux Éditions Eyrolles en 1979 [7], De l'animal à l'Homme aux Éditions du Rocher en 1988 [8] et La Longue Marche du genre humain en 2013 chez L'Harmattan [9].

Il consacre une partie de son temps à la diffusion des connaissances par des conférences à destination de tous les publics, par sa participation à des expositions comme Les Origines de l'Homme inaugurée en 1976 ou celle du parcours permanent de l'ancien musée de l'Homme. Il s'engage dans la création de musées consacrés à l'homme de Neandertal, qui rencontrent toujours un très vif succès, avec « le Préhisto Parc » de Tursac et le musée de l'Homme de Neandertal à La Chapelle-aux-Saints, dont il fut le directeur scientifique. L'association à l'origine du Musée avait choisi de l'honorer en donnant son nom à la salle principale où est exposée la reconstitution de la célèbre sépulture.

Enseignant au niveau académique, il était toujours disposé à partager ses connaissances dans le domaine de la paléoanthropologie et des Néandertaliens, particulièrement avec ses étudiants qui lui sont tous restés fidèles. Citons Jean Granat (1974) sur le thème de la paléo-odontologie, dont les résultats ont permis de mettre au point une méthode d'estimation de l'âge des Néandertaliens, Anne Dambricourt Malassé sur l'évolution de la mandibule et la base du crâne (1983), Nicolas Buchet (2003) sur l'impact de l'environnement sur le squelette humain, Julie Bouhallier (2006), à la suite de Christine Berge, sur l'évolution de la fonction obstétricale chez les hominoïdes, Mélanie Frelat (2007) sur la variabilité des proportions corporelles humaines ou encore Caroline Souday (2008) sur l'analyse morphométrique des dents dans le genre Homo.

La communauté scientifique retiendra de Jean-Louis Heim sa grande culture scientifique et son profond attachement à l'institution du Muséum et du musée de l'Homme, ainsi que sa modestie et son enthousiasme dans le partage des connaissances. Une page de l'histoire de la paléoanthropologie française se tourne avec sa disparition.

\section{Références}

1. Heim JL (1976) Les hommes fossiles de La Ferrassie. Tome I. Le gisement, les squelettes adultes (crânes et squelettes du tronc). Archives de l'Institut de paléontologie humaine, Mémoire 35, $331 \mathrm{p}$

2. Heim JL (1982) Les hommes fossiles de La Ferrassie. Tome II. Les squelettes adultes (squelette des membres). Archives de l'Institut de paléontologie humaine, Mémoire 38, $272 \mathrm{p}$

3. Heim JL (1982) Les enfants néandertaliens de La Ferrassie. Masson, Paris, $169 \mathrm{p}$

4. Heim JL (1989) Une nouvelle reconstitution du crâne néandertalien de La Chapelle-aux-Saints. Méthodes et résultats. BMSAP 1:95-117 
5. Boë LJ, Heim JL, Honda K, Maeda S (2002) The potential Neandertal vowel space was as large as that of modern humans. J Phonetics 30:465-84

6. Ibrahim BA, Dunand F, Heim JL, et al (2008) Le matériel archéologique et les restes humains de la nécropole d'Aïn el-Labakha (oasis de Kharga). Éditions Cybèle, Paris, 203 p
7. Heim JL (1979) 700000 siècles d'histoire humaine. Eyrolles, Paris, $96 \mathrm{p}$

8. Heim JL (1988) De l'animal à l'Homme. Éditions du Rocher, Paris, $124 \mathrm{p}$

9. Heim JL (2013) La longue marche du genre humain. L'Harmattan, Paris, $176 \mathrm{p}$ 